\title{
On a model for population with age structure
}

\author{
Cătălin Şterbeţi $1^{1, *}$ \\ ${ }^{1}$ University of Craiova, Department of Applied Mathematics \\ Al. I. Cuza 13, Craiova 200585, Dolj, Romania
}

\begin{abstract}
In this paper we study a linear continuous model describing age structure into a dynamics of one sex population, related with the McKendrick model. McKendrick assumes that the female population can be described by a function of two variables, age and time. Using the method of characteristics and Laplace transform, it is possible to find the function representing the number of births in unit time $t$ and the total population size in some particular cases. In the content of some works referring to the behavior of age-structure one sex population is presented the complete model of the Lotka-McKendrick equation given in the system (5) for simple cases. The genesis model is a simple one that works with the Dirac distribution and it is presented in [1]. When the birth modulus is given by the relation (9), we determine the differential-difference equation for the function $B(t)$ which represents the number of births in unit time, given in (3).
\end{abstract}

2010 Mathematics Subject Classification: 37N25, 39A06, 44A10, 92D25.

Keywords: Dynamical systems, Population dynamics, Differential-difference equation, Laplace transform.

\section{Introduction}

Mathematical theories of populations have been used in many context in the last two centuries. They have been discussed in important studies of populations, human populations as well as populations of animals, cells or viruses. Such a study can be found in [3]. Several features of populations can be analyzed. The age structure and growth can be studied by considering birth and death forces acting to change them. In particular, the long time state of the population and its sensitivity to changes in birth and death schedules can be determined. Age is a very important parameter because different ages mean different reproduction, different behaviors and survival capacities.

In the paper [6], A.J. Lotka and F.R.Sharpe were among the first scientists who initiated a model in the structure age population. Also, M.Iannelli has a short but interesting monograph [4], which treats the age-structure population dynamics.For the first time in history, A.G.McKendrick in [5] introduced the age structure into the dynamics of one sex population in 1926. The McKendrick model presumes that the female population can be described in two variables, age and time. We denote by $\rho(a, t)$ the density of individuals of age $a$ at time $t$. The number of individuals with ages between $a$ and $a+\Delta a$ at time $t$ is approximately

\footnotetext{
*e-mail: sterbetiro@yahoo.com
} 
$\rho(a, t) \Delta a$. As a result, the total population at time $\mathrm{t}$ can be approximated by

$$
\sum_{a} \rho(a, t) \Delta a
$$

whose "limit as $\Delta a \rightarrow 0$ " is $\int_{0}^{\infty} \rho(a, t) d a$. We define the total size of population

$$
P(t)=\int_{0}^{\infty} \rho(a, t) d a .
$$

In practice, it is plausible to expect that for suficiently large $a$ we have $\rho(a, t)=0$.

Time and age are connected. For the cohort of members of the population born at the same time, say $c, \frac{d a}{d t}=1$ and $a=t-c$. We will consider that members abandon the population only by dying, and that there exists an age-dependent death rate $\mu(a)$. This leads us to the fact that in the time interval from $t$ to $t+\Delta t$ a fraction $\mu(a) \Delta t$ of the members with ages between $a$ and $a+\Delta a$ at time $t$ die. There are $\rho(a, t) \Delta a$ individuals at time t, with ages between $a$ and $a+\Delta a$. The number of deaths from this age cohort between the times $t$ and $t+\Delta t$ is $\rho(a, t) \Delta a \cdot \mu(a) \Delta t$. The remainder survive at time $t+\Delta t$, with ages between $a+\Delta t$ and $a+\Delta t+\Delta a$. Thus,

$$
\rho(a+\Delta t, t+\Delta t) \Delta a \approx \rho(a, t) \Delta a-\rho(a, t) \mu(a) \Delta a \Delta t .
$$

We divide by $\Delta a \Delta t$ the last relation and gives

$$
\frac{\rho(a+\Delta t, t+\Delta t)-\rho(a, t)}{\Delta t}+\mu(a) \rho(a, t) \approx 0 .
$$

We take now $\Delta t \rightarrow 0$. If we consider that $\rho(a, t)$ is a differentiable function in both variables, we have

$$
\begin{aligned}
\lim _{\Delta t \rightarrow 0} \frac{\rho(a+\Delta t, t+\Delta t)-\rho(a, t)}{\Delta t} & =\lim _{\Delta t \rightarrow 0} \frac{\rho(a+\Delta t, t+\Delta t)-\rho(a, t+\Delta t)}{\Delta t}+\lim _{\Delta t \rightarrow 0} \frac{\rho(a, t+\Delta t)-\rho(a, t)}{\Delta t} \\
& =\lim _{\Delta t \rightarrow 0}\left(\rho_{a}(a, t+\Delta t)+\rho_{t}(a, t)\right) \\
& =\rho_{a}(a, t)+\rho_{t}(a, t) .
\end{aligned}
$$

As a consequence, we obtain the Lotka-McKendrick equation (this equation is also named the von Foerster equation- 1959) because the same equation arises in cellular biology.

$$
\rho_{a}(a, t)+\rho_{t}(a, t)+\mu(a) \rho(a, t)=0 .
$$

The function $\mu(a) \geq 0$ is called the death modulus. If $y(\alpha)$ is the number of individuals starting at age a and survive to age $\alpha$, then

$$
y(\alpha+\Delta \alpha)-y(\alpha) \approx-\mu(\alpha) y(\alpha) \Delta \alpha .
$$

Dividing by $\Delta \alpha$ and taking $\Delta \alpha \rightarrow 0$ we obtain $y^{\prime}(\alpha)=-\mu(\alpha) y(\alpha)$, and it follows

$$
y(a)=y\left(a_{0}\right) e^{-\int_{a_{0}}^{a} \mu(\alpha) d \alpha} .
$$

Hence the probability that a subject of age $a_{0}$ will survive to age $a$ is $e^{-\int_{a_{0}}^{a} \mu(\alpha) d \alpha}$. In particular, the probability of survival from age 0 (birth) to age $a$ is

$$
\pi(a)=e^{-\int_{0}^{a} \mu(\alpha) d \alpha} .
$$


Alongside, we consider that the birth process is determined by a function called the birth modulus, $\beta(a)$. Then $\beta(a) \Delta t$ is the number of descendants born by members with ages between $a$ and $a+\Delta a$ in the time interval from $t$ to $t+\Delta t$. So, the total number of births between time $t$ and $t+\Delta t$ is

$$
\Delta t \sum_{a} \beta(a) \rho(a, t) \Delta a \rightarrow \Delta t \int_{0}^{\infty} \beta(a) \rho(a, t) d a,
$$

when $\Delta a \rightarrow 0$. But this quantity must also be $\rho(0, t) \Delta t$. We obtain the renewal condition

$$
B(t)=\rho(0, t)=\int_{0}^{\infty} \beta(a) \rho(a, t) d a .
$$

We can easily see that if we know $B(t)$, then we can evaluate $\rho(a, t)$ as the number of births at time $t-a$ multiplied by the survival fraction to age $a$ plus the number of survivers from the initial population born before time $t-a$ and surviving to time t. The above triangle of the first quadrant of the $(a, t)$-plane represents members of the population who were already alive at time $t=0$. We specify an initial (at $t=0$ ) age distribution $\rho(a, 0)=\Phi(a)$ and in this way we have a full model consisting of a partial differential equation with two auxiliary conditions. This model is

$$
\begin{array}{ll}
\rho_{a}(a, t)+\rho_{t}(a, t)+\mu(a) \rho(a, t) & =0, \\
\rho(0, t) & =\int_{0}^{\infty} \beta(a) \rho(a, t) d a, \\
\rho(a, 0) & =\Phi(a) .
\end{array}
$$

The solution of the model (4) is carried out using the method of characteristics.

\section{The method of Characteristics}

If we decide to transform the above problem into a more attractive form, we will integrate along characteristics of the partial differential equation. The characteristics are the lines $t=a+c$. The value of the function $\rho$ at a point $(a, t)$ is determined by the values of $\rho$ on the characteristics through $(a, t)$ because a member of the population of age $a$ at time $t$ must have been of age $a-\alpha$ at time $t-\alpha$, where $\alpha$ is arbitrary positive with $\alpha \leq a$ and $\alpha \leq t$. In other words, all the points of a given characteristic $t=a+c$ correspond to the same age cohort. For $t \geq a, \rho(a, t)$ is just the number of survivers to age a of the $\rho(0, t-a)$ members born at time $(t-a)$. Since the fraction surviving to age $a$ is $\pi(a)$, we have $\rho(a, t)=\rho(0, t-a) \pi(a)$. for $t \geq a$. If $t \leq a$, then $\rho(a, t)$ is just the number of survivors to age $a$ of the $\Phi(a-t)$ members who were of age $(a-t)$ at time 0 . The fraction surviving from age $(a-t)$ to age $a$ is $\pi(a) / \pi(a-t)$. Therefore, we have $\rho(a, t)=\Phi(a-t) \pi(a) / \pi(a-t)$ for $t<a$. Thus,

$$
\rho(a, t)= \begin{cases}\rho(0, t-a) \pi(a) & \text { for } \quad t \geq a \\ \Phi(a-t) \pi(a) / \pi(a-t) & \text { for } t<a .\end{cases}
$$

We may write this in terms of $\mu$,

$$
\rho(a, t)= \begin{cases}\rho(0, t-a) e^{-\int_{0}^{a} \mu(\alpha) d \alpha} & \text { for } \quad t \geq a, \\ \Phi(a-t) e^{-\int_{a-t}^{a} \mu(\alpha) d \alpha} & \text { for } \quad t<a .\end{cases}
$$


The function $B(t)=\rho(0, t)$ represents the number of births in unit time t. The problem (4) becomes

$$
\begin{array}{ll}
\rho_{a}(a, t)+\rho_{t}(a, t)+\mu(a) \rho(a, t) & =0, \\
B(t) & =\int_{0}^{\infty} \beta(a) \rho(a, t) d a, \\
\rho(a, 0) & =\Phi(a) .
\end{array}
$$

with the representation

$$
\rho(a, t)=\left\{\begin{array}{lll}
B(t-a) e^{-\int_{0}^{a} \mu(\alpha) d \alpha} & \text { for } \quad t \geq a \\
\Phi(a-t) e^{-\int_{a-t}^{a} \mu(\alpha) d \alpha} & \text { for } \quad t<a
\end{array}\right.
$$

We let $\Psi(t)$ be the rate of births from members who were present in the population at time 0 . From (4) we obtain

$$
\begin{aligned}
\rho(0, t) & =\int_{0}^{\infty} \beta(a) \rho(a, t) d a \\
& =\int_{0}^{t} \beta(a) \rho(0, t-a) e^{-\int_{0}^{a} \mu(\alpha) d \alpha} d a+\int_{t}^{\infty} \beta(a) \Phi(a-t) e^{-\int_{a-t}^{a} \mu(\alpha) d \alpha} d a \\
& =\int_{0}^{t} \beta(a) B(t-a) e^{-\int_{0}^{a} \mu(\alpha) d \alpha} d a+\int_{t}^{\infty} \beta(a) \Phi(a-t) e^{-\int_{a-t}^{a} \mu(\alpha) d \alpha} d a .
\end{aligned}
$$

Now we may evaluate $\Psi$ in terms of the initial age distribution and the birth and death moduli

$$
\Psi(t)=\int_{t}^{\infty} \beta(a) \Phi(a-t) e^{-\int_{a-t}^{a} \mu(\alpha) d \alpha} d a=\int_{0}^{\infty} \beta(t+s) \Phi(s) e^{-\int_{s}^{s+t} \mu(\alpha) d \alpha} d s .
$$

We take into account that $\pi(a)=e^{-\int_{0}^{a} \mu(\alpha) d \alpha}$ and obtain for $B(t)$ a linear Volterra equation of convolution type named the renewal equation

$$
B(t)=\Psi(t)+\int_{0}^{t} \beta(a) \pi(a) B(t-a) d a .
$$

Conversely, if $B(t)$ is o solution of renewal equation, we can obtain the solution of the initial problem given by

$$
\rho(a, t)= \begin{cases}B(t-a) e^{-\int_{0}^{a} \mu(\alpha) d \alpha} & \text { for } \quad t \geq a \\ \Phi(a-t) e^{-\int_{a-t}^{a} \mu(\alpha) d \alpha} & \text { for } \quad t<a\end{cases}
$$

The problem we will study now describes the behaviour of solutions of the renewal equation under the assumptions that $\Phi(t) \rightarrow 0$ as $t \rightarrow \infty, \int_{0}^{\infty} \Phi(t) d t<\infty$ and $R=\int_{0}^{\infty} \beta(a) \pi(a) d a<$ $\infty$. Here $R$ is the expected number of offspring for each individual over a lifetime, being the sum over all ages $a$ of probability of survival to age $a$ multiplied by the number of offspring at age $a$. 
One approach of this problem involves taking Laplace transforms and using the convolution theorem in the renewal equation to obtain

$$
B_{1}(p)=\Psi_{1}(p)+F_{1}(p) B_{1}(p),
$$

where $B_{1}, \Psi_{1}$ and $F_{1}$ are the Laplace transforms of $B, \Psi$ and $\beta \pi$. Taking into account that, in the mentioned conditions, there is a unique real solution $p_{0}$ with $F_{1}\left(p_{0}\right)=1$, in [1] it is shown that

$$
B(t)=B e^{p_{0} t}+E(t)
$$

where $|E(t)| \leq c e^{\left(p_{0}-\varepsilon\right) t}$, as $t \rightarrow 0$. From the representation of $\rho(a, t)$ in terms of $B(t)$ we obtain

$$
\rho(a, t)=B e^{p_{0}(t-a)} \pi(a)+E(t-a),
$$

and thus

$$
P(t)=P e^{p_{0} t}+E(t)
$$

for some constant $P$ and a function $E(t)$ that grows no faster than $e^{\left(p_{0}-\varepsilon\right) t}$.

\section{Main result}

In the following, we consider a model with $\Phi(a)$ arbitrary, $\mu$ positive constant function and

$$
\beta(a)=\left\{\begin{array}{lll}
\beta & \text { for } & a \leq T \\
\beta e^{-\mu a} & \text { for } & a>T
\end{array}\right.
$$

We shall express the differential-difference equation for the function $B(t)$ in both cases $t<T$ and $t>T$.

Theorem 3.1 For $t<T$, the function $B$ is solution of the equation

$$
B^{\prime \prime}(t)-(\beta-3 \mu) B^{\prime}(t)+2 \mu(\mu-\beta) B(t)=-\mu \beta e^{-\mu t} \Phi(T-t)+\beta e^{-\mu t}\left(1-e^{-\mu T}\right) \Phi^{\prime}(T-t),
$$

where $B(0)=\int_{0}^{T} \beta \Phi(a) d a+\int_{T}^{\infty} \beta e^{-\mu a} \Phi(a) d a$.

Proof. The formula for $B(0)$ follows immediately from the renewal equation. Taking into account that

$$
\pi(a)=e^{-\int_{0}^{a} \mu d \alpha}=e^{-\mu a}
$$

and that $B(t)$ is a solution of the renewal equation,

$$
B(t)=\Psi(t)+\int_{0}^{t} \beta(a) \pi(a) B(t-a) d a=\Psi(t)+\int_{0}^{t} \beta(a) e^{-\mu a} B(t-a) d a
$$

where

$$
\Psi(t)=\int_{t}^{\infty} \beta(a) \Phi(a-t) e^{-\int_{a-t}^{a} \mu(\alpha) d \alpha} d a=\int_{0}^{\infty} \beta(t+s) \Phi(s) e^{-\mu t} d s
$$


for $t<T$, we obtain

$$
\begin{aligned}
B(t) & =\int_{0}^{T-t} \beta(t+s) \Phi(s) e^{-\mu t} d s+\int_{T-t}^{\infty} \beta(t+s) \Phi(s) e^{-\mu t} d s+\int_{0}^{t} \beta(a) e^{-\mu a} B(t-a) d a \\
& =\beta e^{-\mu t} \int_{0}^{T-t} \Phi(s) d s+\beta \int_{T-t}^{\infty} e^{-\mu(t+s)} \Phi(s) e^{-\mu t} d s+\int_{0}^{t} \beta e^{-\mu a} B(t-a) d a \\
& =\beta e^{-\mu t} \int_{0}^{T-t} \Phi(s) d s+\beta e^{-2 \mu t} \int_{T-t}^{\infty} e^{-\mu s} \Phi(s) d s+\beta \int_{0}^{t} e^{-\mu a} B(t-a) d a .
\end{aligned}
$$

We differentiate the last equation and obtain

$$
\begin{aligned}
B^{\prime}(t) & =-\beta \mu e^{-\mu t} \int_{0}^{T-t} \Phi(s) d s-\beta e^{-\mu t} \Phi(T-t)-2 \mu \beta e^{-2 \mu t} \int_{T-t}^{\infty} e^{-\mu s} \Phi(s) d s \\
& +\beta e^{-2 \mu t} e^{-\mu(T-t) \Phi(T-t)}+\beta \int_{0}^{t} e^{-\mu a} B_{t}^{\prime}(t-a) d a+\beta e^{-\mu t} B(0) \\
& =-\beta \mu e^{-\mu t} \int_{0}^{T-t} \Phi(s) d s-\beta e^{-\mu t} \Phi(T-t)-2 \mu \beta e^{-2 \mu t} \int_{T-t}^{\infty} e^{-\mu s} \Phi(s) d s \\
& +\beta e^{-\mu(t+T)} \Phi(T-t)+\beta\left[-\left.e^{-\mu a} B(t-a)\right|_{a=0} ^{a=t}+e^{-\mu t} B(0)-\int_{0}^{t} \mu e^{-\mu a} B(t-a) d a\right] \\
& =-\beta \mu e^{-\mu t} \int_{0}^{T-t} \Phi(s) d s-\beta e^{-\mu t} \Phi(T-t)-2 \mu \beta e^{-2 \mu t} \int_{T-t}^{\infty} e^{-\mu s} \Phi(s) d s \\
& +\beta e^{-\mu(t+T)} \Phi(T-t)+\beta\left[-e^{-\mu t} B(0)+B(t)-\mu \int_{0}^{t} e^{-\mu a} B(t-a) d a+e^{-\mu t} B(0)\right] \\
& =-\beta e^{-\mu t} \Phi(T-t)+\beta e^{-\mu(t+T)} \Phi(T-t)+\beta B(t)-\mu \beta e^{-2 \mu t} \int_{T-t}^{\infty} e^{-\mu s} \Phi(s) d s \\
& -\mu\left[\beta e^{-\mu t} \int_{0}^{T-t} \Phi(s) d s+\beta e^{-2 \mu t} \int_{T-t}^{\infty} e^{-\mu s} \Phi(s) d s+\beta \int_{0}^{t} e^{-\mu a} B(t-a) d a\right] .
\end{aligned}
$$

Taking into account that in the square brackets of the last equality we have the value of $B(t)$, we find that

$$
B^{\prime}(t)=-\beta e^{-\mu t} \Phi(T-t)+\beta e^{-\mu(t+T)} \Phi(T-t)+\beta B(t)-\mu B(t)-\mu \beta e^{-2 \mu t} \int_{T-t}^{\infty} e^{-\mu s} \Phi(s) d s .
$$

By differentiating, we get

$$
\begin{aligned}
B^{\prime \prime}(t) & =\mu \beta e^{-\mu t}\left(1-e^{-\mu T}\right) \Phi(T-t)+\beta e^{-\mu t}\left(1-e^{-\mu T}\right) \Phi^{\prime}(T-t)+(\beta-\mu) B^{\prime}(t) \\
& +2 \mu^{2} \beta e^{-2 \mu t} \int_{T-t}^{\infty} e^{-\mu s} \Phi(s) d s-\mu \beta e^{-2 \mu t} e^{-\mu(T-t)} \Phi(T-t) \\
& =\mu \beta e^{-\mu t} \Phi(T-t)-\mu \beta e^{-\mu(t+T)} \Phi(T-t)+\beta e^{-\mu t}\left(1-e^{-\mu T}\right) \Phi^{\prime}(T-t)+(\beta-\mu) B^{\prime}(t) \\
& -\mu \beta e^{-\mu(t+T)} \Phi(T-t)-2 \mu\left[B^{\prime}(t)+\beta e^{-\mu t}\left(1+e^{-\mu T}\right) \Phi(T-t)+(\mu-\beta) B(t)\right] \\
& =-\mu \beta e^{-\mu t} \Phi(T-t)+\beta e^{-\mu t}\left(1-e^{-\mu T}\right) \Phi^{\prime}(T-t)+(\beta-3 \mu) B^{\prime}(t)-2 \mu(\mu-\beta) B(t) .
\end{aligned}
$$

We obtain the differential equation of second order for $B(t)$

$$
B^{\prime \prime}(t)-(\beta-3 \mu) B^{\prime}(t)+2 \mu(\mu-\beta) B(t)=-\mu \beta e^{-\mu t} \Phi(T-t)+\beta e^{-\mu t}\left(1-e^{-\mu T}\right) \Phi^{\prime}(T-t),
$$

and the Theorem 1 is proved. 
Theorem 3.2 For $t \geq T$, the function $B$ is solution of the following equation

$$
B^{\prime \prime}(t)=(\beta-3 \mu) B^{\prime}(t)+\beta\left(e^{-2 \mu T}-e^{-\mu T}\right) B^{\prime}(t-T)+2 \mu(\beta-\mu) B(t)+\mu \beta\left(e^{-2 \mu T}-e^{-\mu T}\right) B(t-T) .
$$

Proof. For $t \geq T$, we can write

$$
B(t)=\Psi(t)+\int_{0}^{t} \beta(a) e^{-\mu a} B(t-a) d a
$$

Here we have

$$
\Psi(t)=\int_{t}^{\infty} \beta(a) \Phi(a-t) e^{-\int_{a-t}^{a} \mu d \alpha} d a=\int_{t}^{\infty} \beta e^{-\mu a} \Phi(a-t) e^{-\mu t} d a=\beta e^{-\mu t} \int_{t}^{\infty} e^{-\mu a} \Phi(a-t) d a .
$$

and

$$
\int_{0}^{t} \beta(a) e^{-\mu a} B(t-a) d a=\int_{0}^{T} \beta e^{-\mu a} B(t-a) d a+\int_{T}^{t} \beta e^{-2 \mu a} B(t-a) d a .
$$

But

$$
\begin{aligned}
\Psi^{\prime}(t) & =-\beta \mu e^{-\mu t} \int_{t}^{\infty} e^{-\mu a} \Phi(a-t) d a+\beta e^{-\mu t} \frac{d}{d t}\left[\int_{t}^{\infty} e^{-\mu a} \Phi(a-t) d a\right] \\
& =-\mu \Psi(t)-\beta e^{-\mu t}\left[\int_{t}^{\infty} e^{-\mu a} \Phi^{\prime}(a-t) d a-e^{-\mu t} \Phi(0)\right] \\
& =-\mu \Psi(t)-\beta e^{-\mu t}\left[\left.e^{-\mu a} \Phi(a-t)\right|_{a=t} ^{a=\infty}+\int_{t}^{\infty} \mu e^{-\mu a} \Phi(a-t) d a+e^{-\mu t} \Phi(0)\right] \\
& =-\mu \Psi(t)-\mu \beta e^{-\mu t} \int_{t}^{\infty} e^{-\mu a} \Phi(a-t) d a=-2 \mu \Psi(t) .
\end{aligned}
$$

under the additional assumption that $\lim _{a \rightarrow \infty} e^{-\mu a} \Phi(a)=0$.

We conclude that $\Psi^{\prime}(t)=-2 \mu \Psi(t)$. Moreover,

$$
\int_{0}^{t} \beta(a) e^{-\mu a} B(t-a) d a=\int_{0}^{T} \beta e^{-\mu a} B(t-a) d a+\int_{T}^{t} \beta e^{-2 \mu a} B(t-a) d a .
$$

We can calculate the derivatives of the last two terms in the right-hand side of the last equality and obtain

$$
\begin{aligned}
\frac{d}{d t}\left[\int_{0}^{T} \beta e^{-\mu a} B(t-a) d a\right] & =\int_{0}^{T} \beta e^{-\mu a} B^{\prime}(t-a) d a \\
& =-\left.\beta e^{-\mu a} B(t-a)\right|_{a=0} ^{a=T}+\int_{0}^{T} \beta(-\mu) e^{-\mu a} B(t-a) d a \\
& =-\beta e^{-\mu T} B(t-T)+\beta B(t)-\mu \beta \int_{0}^{T} e^{-\mu a} B(t-a) d a
\end{aligned}
$$


and

$$
\begin{aligned}
\frac{d}{d t}\left[\int_{T}^{t} \beta e^{-2 \mu a} B(t-a) d a\right] & =\int_{T}^{t} \beta e^{-2 \mu a} B^{\prime}(t-a) d a+\beta e^{-2 \mu t} B(0) \\
& =-\left.\beta e^{-2 \mu a} B(t-a)\right|_{a=T} ^{a=t}-\beta \int_{T}^{t} 2 \mu e^{-2 \mu a} B(t-a) d a+\beta e^{-2 \mu t} B(0) \\
& =-\beta e^{-2 \mu t} B(0)+\beta e^{-2 \mu T} B(t-T)-2 \mu \int_{T}^{t} \beta e^{-2 \mu a} B(t-a) d a+\beta e^{-2 \mu t} B(0) \\
& =\beta e^{-2 \mu T} B(t-T)-2 \mu \int_{T}^{t} \beta e^{-2 \mu a} B(t-a) d a .
\end{aligned}
$$

We add the last two relations and we get

$$
\begin{aligned}
\frac{d}{d t}\left[\int_{0}^{t} \beta(a) \pi(a) B(t-a) d a\right] & =-\beta e^{-\mu T} B(t-T)+\beta B(t)+\beta e^{-2 \mu T} B(t-T) \\
& -\mu \beta \int_{0}^{T} e^{-\mu a} B(t-a) d a-2 \mu \beta \int_{T}^{t} e^{-2 \mu a} B(t-a) d a \\
& =\beta B(t)+\beta\left(e^{-2 \mu T}-e^{-\mu T}\right) B(t-T)+\mu \beta \int_{0}^{T} e^{-\mu a} B(t-a) d a \\
& -2 \mu\left[\beta \int_{0}^{t} e^{-\mu a} B(t-a) d a+\beta \int_{T}^{t} e^{-2 \mu a} B(t-a) d a\right] \\
& =\beta\left[B(t)+\left(e^{-2 \mu T}-e^{-\mu T}\right) B(t-T)\right]+\mu \beta \int_{0}^{T} e^{-\mu a} B(t-a) d a \\
& -2 \mu \int_{0}^{t} \beta(a) \pi(a) B(t-a) d a .
\end{aligned}
$$

We use also that $\Psi^{\prime}(t)=-2 \mu \Psi(t)$ and we conclude

$$
\begin{aligned}
B^{\prime}(t) & =\Psi^{\prime}(t)+\frac{d}{d t}\left[\int_{0}^{t} \beta(a) \pi(a) B(t-a) d a\right] \\
& =-2 \mu \Psi(t)-2 \mu \int_{0}^{t} \beta(a) \pi(a) B(t-a) d a \\
& +\beta\left[B(t)+\left(e^{-2 \mu T}-e^{-\mu T}\right) B(t-T)\right]+\mu \beta \int_{0}^{T} e^{-\mu a} B(t-a) d a .
\end{aligned}
$$

Thus, we have

$$
B^{\prime}(t)=(\beta-2 \mu) B(t)+\beta\left(e^{-2 \mu T}-e^{-\mu T}\right) B(t-T)+\mu \beta \int_{0}^{T} e^{-\mu a} B(t-a) d a .
$$

By differentiating in the last equality, we find

$$
\begin{aligned}
B^{\prime \prime}(t) & =(\beta-2 \mu) B^{\prime}(t)+\beta\left(e^{-2 \mu T}-e^{-\mu T}\right) B^{\prime}(t-T) \\
& +\mu \beta\left[-e^{-\mu t} B(t-T)+B(t)\right]+\mu\left[-\mu \beta \int_{0}^{T} e^{-\mu a} B(t-a) d a\right] \\
& =(\beta-2 \mu) B^{\prime}(t)+\beta\left(e^{-2 \mu T}-e^{-\mu T}\right) B^{\prime}(t-T) \\
& +\mu \beta\left[-e^{-\mu t} B(t-T)+B(t)\right]+\mu\left[(\beta-2 \mu) B(t)+\beta\left(e^{-2 \mu T}-e^{-\mu T}\right) B(t-T)-B^{\prime}(t)\right] .
\end{aligned}
$$


We deduce that $B(t)$ satisfies the differential-difference equation

$$
B^{\prime \prime}(t)=(\beta-3 \mu) B^{\prime}(t)+\beta\left(e^{-2 \mu T}-e^{-\mu T}\right) B^{\prime}(t-T)+2 \mu(\beta-\mu) B(t)+\mu \beta\left(e^{-2 \mu T}-e^{-\mu T}\right) B(t-T) .
$$

and the equation from (11) in Theorem 2 is proved.

\section{Conclusions}

1) We used several times in the proof of both theorems the following formula

$$
\frac{d}{d t} \int_{u(t)}^{v(t)} f(a, t) d a=\int_{u(t)}^{v(t)} \frac{\partial f}{\partial t}(a, t) d a+f(v(t), t) v^{\prime}(t)-f(u(t), t) u^{\prime}(t)
$$

available if $u$ and $v$ are differentiable, $f$ is continuous in both variables and $f$ has partial derivatives with respect to $t$ with $\frac{\partial f}{\partial t}$ continuous.

2) The case $T=0$ leads us to the more particular model proposed by F. Brauer and C.Castillo-Chavez in [1] at p.281, with $\mu$ constant, $\Phi(a)$ arbitrary and $\beta(a)=\beta e^{-\mu a}$. For this model, $B^{\prime}(t)=(\beta-2 \mu) B(t)$ and $B(0)=\beta \int_{0}^{\infty} e^{-\mu s} \Phi(s) d s$, as we can easily see from (12) with the mentioned function $\beta$.

3 ) If we also know the function $\Phi$, from the equations (10) and (11), we can write and solve the differential equations for $B$ in the cases $t<T$ and $t \geq T$. In this way we can express a formula for the density of population $\rho$. After integration with respect to $a$, we obtain the total population size.

\section{Acknowledgments}

This research was supported by Horizon2020-2017-RISE-777911 project.

\section{References}

[1] F. Brauer, and C. Castillo-Chavez, Mathematical Models in Population Biology and Epidemiology, Second Edition (Texts in Applied Mathematics 40, Springer, New York, 2012) $532 \mathrm{pp}$.

[2] F. Brauer, and C. Castillo-Chavez and Z. Feng Mathematical Models in Epidemiology, Second Edition (Texts in Applied Mathematics 69, New York, 2019) 637 pp.

[3] F.C. Hoppensteadt, Mathematical Theories of Populations: Demographics, Genetics and Epidemics (SIAM, Philadelphia, 1975) 79 pp.

[4] M. Iannelli, Mathematical Theory of Age-Structured Population Dynamics (Appl. Mathematical Monographs, C.N.R., 1995) 174 pp.

[5] A.G. McKendrick, Applications of mathematics to medical problems, Proc. Edinburgh Math. Soc. 44, 98-130 (1926).

[6] F.R. Sharpe, and A.J. Lotka, A problem in age distribution, The London, Edinburgh and Dublin Philosophical Magazine and Journal of Science 21(124), 435-438 (1911). 